\title{
Baf60b-mediated ATM-p53 activation blocks cell identity conversion by sensing chromatin opening
}

Shuyi $\mathrm{Ji}^{1,}{ }^{1,}$, Linying Zhu ${ }^{1, *}$, Yimeng Gao ${ }^{1}$, Xiaoran Zhang ${ }^{2}$, Yupeng Yan $^{1}$, Jin $\mathrm{Cen}^{1}$, Rongxia $\mathrm{Li}^{3}$, Rong Zeng ${ }^{3}$, Lujian Liao ${ }^{4}$, Chunhui $\mathrm{Hou}^{5}$, Yawei Gao ${ }^{6}$, Shaorong $\mathrm{Gao}^{6}$, Gang $\mathrm{Wei}^{2}$, Lijian Hui ${ }^{1,7}$

${ }^{I}$ State Key Laboratory of Cell Biology, Institute of Biochemistry and Cell Biology, Shanghai Institutes for Biological Sciences, Chinese Academy of Sciences, Shanghai 200031, China; ${ }^{2}$ CAS Key Laboratory of Computational Biology, CAS-MPG Partner Institute for Computational Biology, Shanghai Institutes for Biological Sciences, Chinese Academy of Sciences, Shanghai 200031, China; ${ }^{3}$ Key Laboratory of Systems Biology, Institute of Biochemistry and Cell Biology, Shanghai Institutes for Biological Sciences, Chinese Academy of Sciences, Shanghai 200031, China; ${ }^{4}$ Shanghai Key Laboratory of Regulatory Biology, School of Life Sciences, East China Normal University, Shanghai 200241, China; ${ }^{5}$ Department of Biology, South University of Science and Technology of China, Shenzhen, Guangdong 518055, China $;{ }^{6}$ Clinical and Translational Research Center of Shanghai First Maternity and Infant Hospital, Shanghai Key Laboratory of Signaling and Disease Research, School of Life Sciences and Technology, Tongji University, Shanghai 200092, China; ${ }^{7}$ School of Life Science and Technology, Shanghai Tech University, 100 Haike Road, Shanghai 201210, China

Lineage conversion by expression of lineage-specific transcription factors is a process of epigenetic remodeling that has low efficiency. The mechanism by which a cell resists lineage conversion is largely unknown. Using hepatic-specific transcription factors Foxa3, Hnf1 $\alpha$ and Gata4 (3TF) to induce hepatic conversion in mouse fibroblasts, we showed that 3TF induced strong activation of the ATM-p53 pathway, which led to proliferation arrest and cell death, and it further prevented hepatic conversion. Notably, ATM activation, independent of DNA damage, responded to chromatin opening during hepatic conversion. By characterizing the early molecular events during hepatic conversion, we found that Baf60b, a member of the SWI/SNF chromatin remodeling complex, links chromatin opening to ATM activation by facilitating ATM recruitment to the open chromatin regions of a panel of hepatic gene loci. These findings shed light on cellular responses to lineage conversion by revealing a function of the ATM-p53 pathway in sensing chromatin opening.

Keywords: Baf60b; ATM; p53; chromatin remodeling; lineage conversion; hepatic conversion Cell Research (2017) 27:642-656. doi:10.1038/cr.2017.36; published online 17 March 2017

\section{Introduction}

In mammals, the identity of a differentiated cell, shaped by epigenetic and extracellular cues, is stably maintained for tissue homeostasis [1-3]. During development and tissue regeneration, stem cells are committed to their differentiated identity. The stability of the differentiated identity of cells is essential for the growth, survival and perpetuation of all multicellular organisms.

\footnotetext{
*These two authors contributed equally to this work. Correspondence: Lijian Hui ${ }^{\mathrm{a}}$, Gang Wei ${ }^{\mathrm{b}}$

a'E-mail: ljhui@sibcb.ac.cn

bE-mail: weigang@picb.ac.cn

Received 21 January 2017; revised 7 February 2017; accepted 10 February 2017; published online 17 March 2017
}

Recent findings have demonstrated remarkable plasticity of cell identity in mammals, e.g., in vitro lineage conversion induced by forced expression of lineage-specific transcription factors [4-8]. Reprogramming of somatic cells to induced pluripotent stem (iPS) cells was achieved by the ectopic expression of Oct4, Sox2, Klf4 and c-Myc. The use of lineage-specific transcription factors was also applied to the induction of neuronal cells, cardiomyocyte-like cells and hepatocyte-like cells [9-13]. Because the culture medium conditions are well defined in these experimental systems, cell identity conversion thus shown is mainly controlled by the network of lineage-specific transcription factors. In addition, cell identity conversion induced by transcription factor demonstrates that the epigenetic modifications of a differentiat- 
ed cell are plastic and subjected to reprogramming.

Notably, in vitro lineage conversion is often a low-efficiency process. It was proposed that there is a "barrier" against lineage conversion, which was largely discussed at the epigenetic level [4-8]. However, the molecular basis of the "barrier" remains largely elusive. Specifically, given the importance to maintain cell identity and the plasticity of epigenetic modifications, it is interesting to ask whether there is an essential cellular mechanism beyond the epigenetic "barrier" that senses cell identity change and consequently blocks the process $[12,14]$. We approached this question by characterizing Foxa3, Hnfl $\alpha$ and Gata4 (3TF)-induced hepatic conversion in mouse fibroblasts [12].

\section{Results}

Transcription factor-induced ATM and p53 activation impedes hepatic lineage conversion

Wild-type (WT) tail-tip fibroblasts (TTFs) underwent a prominent proliferation arrest and cell death after $3 \mathrm{TF}$ transduction, which largely restrained hepatic conversion (Figure 1A and 1B and Supplementary information, Figure S1A). Our previous study showed that p19Arf inactivation facilitates induced hepatic (iHep) cell formation $[12,14]$. Because p19Arf is a key regulator of the $\mathrm{p} 53$ pathway $[15,16]$, we asked whether $\mathrm{p} 53$ acted as a roadblock to hepatic conversion. As determined by western blot analysis, we found that phosphorylated p53 (p-p53) and total p53 protein levels were increased after $3 \mathrm{TF}$ transduction (Figure 1C). In addition, expression levels of p53 target genes, including p19Arf, p21, Noxa, Puma, Bax and $M d m 2$ [15, 16], were induced (Figure $1 C$ and $1 \mathrm{D})$, suggesting that the $\mathrm{p} 53$ pathway is activated. Importantly, upon deletion of p53, 3TF-induced proliferation arrest and cell death were reversed (Supplementary information, Figure S1B and S1C). Although only 2-3 iHep colonies were obtained from $10^{5}$ WT cells, iHep formation was drastically increased in p53-knockout cells (Figure 1E and Supplementary information, Figure S1D and S1E). Moreover, the generation of iHep cells was improved by $\mathrm{p} 53$ inactivation using $p 53^{+/-}$heterozygous cells (Figure 1E) or short hairpin RNA (shRNA)-mediated p53 silencing (Supplementary information, Figure $\mathrm{S} 1 \mathrm{~F}$ and $\mathrm{S} 1 \mathrm{G})$. These results showed that $\mathrm{p} 53$ is a barrier against hepatic conversion.

Interestingly, p53 has been previously shown to limit conversions between cell identities, including iPS generation [17-21] and oncogenic transformation [22, 23]. We next characterized the mechanism by which p53 is activated during hepatic conversion. We did not find changes in c-Myc and Ras expression (Supplementary informa- tion, Figure S2A), Ras GTPase activity (Supplementary information, Figure S2B), p38 $\alpha$ phosphorylation (Supplementary information, Figure S2C and S2D) or reactive oxygen species levels (Supplementary information, Figure S2E), which are major causes of p53 activation $[15,16]$. In contrast, extensive ATM phosphorylation was observed after $3 \mathrm{TF}$ transduction (Figure $1 \mathrm{~F}$ and $1 \mathrm{G}$ and Supplementary information, Figure S3A). Because ATM deletion was toxic to TTFs (data not shown), we used $A T M^{+/-}$cells to characterize the function of ATM in hepatic conversion. $A T M^{+/}$cells showed attenuated p53 phosphorylation (Figure $1 \mathrm{H}$ ) and increased iHep cell formation (Figure 1I and Supplementary information, Figure S3B and S3C). KU55933, an ATM-specific inhibitor, was used to block ATM activation at the beginning of iHep induction. Phosphorylation of $\mathrm{H} 2 \mathrm{AX}(\gamma \mathrm{H} 2 \mathrm{AX})$, a major downstream substrate of ATM [24], was reduced after KU55933 treatment. In addition, KU55933 decreased p53 phosphorylation levels and facilitated formation of iHep cells (Supplementary information, Figure S3D and S3E). Intriguingly, in $A T M^{+/-}$, KU55933-treated and $p 53^{+/-}$iHep cells, hepatic genes were expressed at levels comparable to those in WT iHep cells (Supplementary information, Figures S3C, S3F and S1E). These results indicate that activation of the ATM-p53 pathway blocks hepatic conversion without interfering with hepatic gene induction.

We next asked whether hepatic conversion might induce extensive DNA damage, which would result in highly activated ATM [25]. DNA strand breaks were undetectable by the comet assay after 3TF transduction [26] (Supplementary information, Figure S4A). In contrast, comet tails were readily detected after 1 Gy $\gamma$-irradiation, which triggered p-ATM at a level lower than that in iHep induction (Supplementary information, Figure S4A). DNA strand breaks were also not detected by the TUNEL assay (Supplementary information, Figure S4B). RPA and 53BP1, key players in DNA damage repair [27], were not activated after $3 \mathrm{TF}$ transduction as shown by immunofluorescence staining (Supplementary information, Figure S4C and S4D). Furthermore, genes involved in double-strand break repair, nucleotide excision repair and base excision repair [27] were not upregulated after $3 \mathrm{TF}$ transduction (Supplementary information, Figure S4E). Nbs1 and ATMIN activate ATM in a DNA damage-dependent and -independent manner, respectively [28-30]. Interestingly, shRNA-mediated silencing of ATMIN, but not Nbs1, attenuated 3TF-induced ATM and p53 activation (Supplementary information, Figure S5AS5D). Furthermore, ATMIN knockdown enhanced the generation of iHep cells (Supplementary information, Figure S5E), suggesting ATMIN-dependent phosphory- 
A
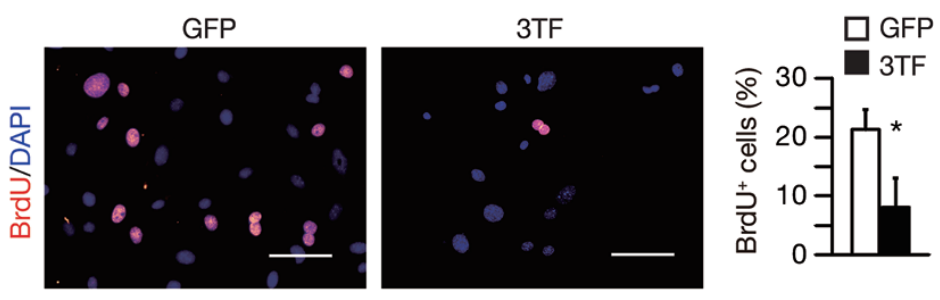

C

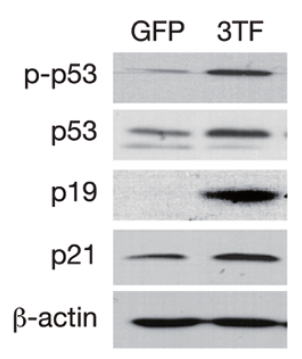

D

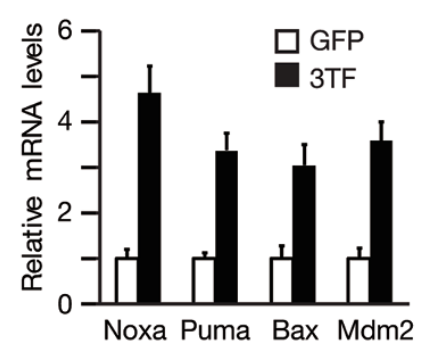

B

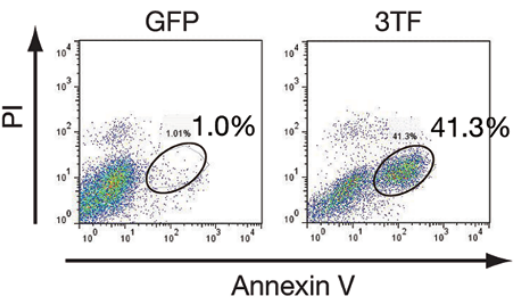

E



G

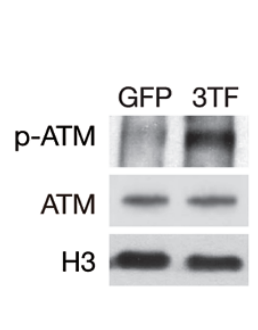

$\mathbf{F}$

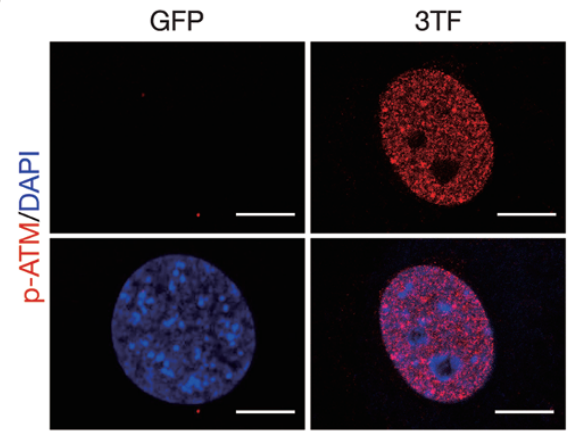

H

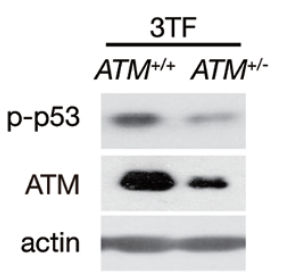

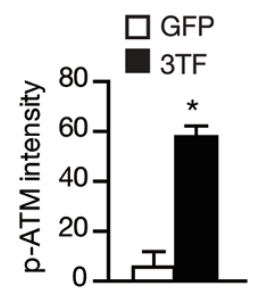

Figure 1 ATM and p53 impede hepatic conversion. (A) 3TF-induced proliferation arrest in wild-type (WT) tail-tip fibroblasts (TTFs) was determined by BrdU incorporation and staining at day 3. GFP expression vector was used as a control. BrdU-positive cells were quantified, $n=6$ fields. Scale bars, $100 \mu \mathrm{m}$. (B) 3TF-induced cell death was analyzed by Annexin $\mathrm{V}$ and propidium iodide (PI) staining at day 6. (C) Levels of phosphorylated p53 (p-p53, Ser15), p19 and p21 were characterized by western blot analyses. (D) mRNA levels of p53 target genes were quantified by qRT-PCR. (E) iHep cells were generated from WT, $p 53^{-/-}, p 53^{+/-}$and $p 19^{-/-}$TTFs by 3TF transduction. iHep colonies were quantified 8 days after 3 TF transduction, $n=4$ independent experiments. (F) Elevated level of ATM phosphorylation at Ser1981 (p-ATM) was determined by immunofluorescence staining $48 \mathrm{~h}$ after 3TF transduction. The fluorescent intensity was quantified by LAS AF Lite software. Analyzed cell numbers: $n=32$ for GFP group and $n=33$ for 3TF group. Scale bars, $10 \mu \mathrm{m}$. (G-H) p-ATM (G) and p-p53 (H) levels were analyzed by the western blot assay in WT TTFs (G) or ATM $^{++}$and ATM $^{+-}(\mathbf{H})$ TTFs $48 \mathrm{~h}$ after $3 T F$ transduction. (I) Quantification of iHep colonies generated from $A T M^{+/+}$and $A T M^{+/-}$TTFs. $n=3$ for each group. Error bars indicate SD. ${ }^{*} P<0.05$, Student's $t$-test. 
lation of ATM. These findings indicate that it is unlikely that DNA damage, if any, causes highly phosphorylated levels of ATM during hepatic conversion.

Early molecular events during hepatic lineage conversion

To uncover the mechanism underlying ATM activation, we analyzed 3TF-induced molecular changes. We first characterized the regulation of the Albumin and $H n f 4 \alpha$ genes, both of which are prominently induced by 3TF and are critical for the establishment of hepatic identity $[12,13,31]$. Twelve hours after transduction, 3TF were already expressed at levels comparable to those in liver cells (Figure 2A). Because Foxa3 and Gata4 act as pioneer factors to open closed chromatin during liver development $[32,33]$, we determined the chromatin opening after $3 \mathrm{TF}$ induction. Chromatin immunoprecipitation (ChIP) assays showed that $3 \mathrm{TF}$ bound upstream regulatory regions of the Albumin and $H n f 4 \alpha$ transcriptional starting sites (Figure 2B and Supplementary information, Figure S6A). As a result of 3TF binding, the upstream regulatory regions of the Albumin and $H n f 4 \alpha$ genes were opened at $12 \mathrm{~h}$ after $3 \mathrm{TF}$ transduction as determined by the micrococcal nuclease digestion assay (Figure 2C), suggesting that chromatin opening happens early during hepatic conversion. Marks of active genome regions, such as $\mathrm{H} 3 \mathrm{~K} 9 \mathrm{ac}$ and $\mathrm{H} 3 \mathrm{~K} 4 \mathrm{me} 2$, were also increased at the upstream regulatory regions of the Albumin and $\mathrm{Hn}$ $f 4 \alpha$ genes mainly at $48 \mathrm{~h}$ after $3 \mathrm{TF}$ transduction (Figure 2D and 2E and Supplementary information, Figure S6B). Finally, the mRNA levels of hepatic genes, including Albumin and $H n f 4 \alpha$, were induced at $72 \mathrm{~h}$ (Figure 2F). These data collectively revealed sequential molecular events after 3TF transduction.

Kinetic analyses of ATM and p53 phosphorylation showed that both ATM and p53 were activated as early as $24 \mathrm{~h}$ after $3 \mathrm{TF}$ transduction (Figure 2G and Supplementary information, Figure S7A-S7C). These timecourse analyses indicated a temporal correlation between chromatin opening and ATM activation. Markedly, we found that p-ATM was recruited to open chromatin regions of the Albumin and Hnf4 $\alpha$ genes after 3TF transduction (Figure 2H). Furthermore, 3TF-induced chromatin opening, H3K9 acetylation and p-ATM binding were validated on a panel of hepatic genes (Supplementary information, Figure S7D-S7F and Table S3). These results suggest that ATM activation is a molecular consequence associated with chromatin opening at a panel of hepatic gene loci.

\section{Baf60b is required for 3TF-induced ATM activation}

We next characterized the connection between chromatin opening and ATM activation, and we sought to identify molecules mediating these two events. Chromatin remodeling complexes are the main regulatory complexes that open chromatin and allow DNA and histones to be accessible $[34,35]$. We silenced the expression of components of the SWI/SNF, CHD, INO80 and ISWI chromatin remodeling complexes by specific shRNAs (Supplementary information, Figure S8A). These shRNAs efficiently decreased the mRNA levels of target genes as determined by qRT-PCR (Supplementary information, Figure S8A). Knockdown of Baf60b and Mta1 reduced the activation of ATM after 3 TF transduction (Figure 3A and Supplementary information, Figure S8B and S8C). Intriguingly, Mta1 knockdown significantly reduced cell number (Supplementary information, Figure S8D and S8E), suggesting that Mta1 silencing may have an adverse role in hepatic conversion. In contrast, depletion of Baf60b, a member of the SWI/SNF complex, markedly increased the generation of iHep colonies (Figure 3B). We confirmed the function of Baf60b by additional shRNAs that target Baf60b CDS (sh1-Baf60b) and 3UTR (sh2-Baf60b) (Supplementary information, Figure S8F). Both shRNAs significantly reduced p-ATM and p-p53 levels (Figure 3C and 3D and Supplementary information, Figure S8G). Baf60b knockdown alleviated proliferation arrest during hepatic conversion and eventually enhanced iHep formation (Figure $3 \mathrm{E}$ and $3 \mathrm{~F}$ and Supplementary information, Figure S9A and S9B). Similar results were found using CRISPR/Cas9-mediated Baf60b-knockout cells (Supplementary information, Figure S9C-S9E). Notably, Bafb0b-silenced iHep cells showed comparable hepatic functions with control iHep cells, including albumin secretion, glycogen storage and drug metabolism (Supplementary information, Figure S9F and S9G), and they appeared to be non-tumorigenic (Supplementary information, Figure S9H). These findings demonstrate a regulatory role of Baf60b in ATM activation and iHep formation.

\section{Baf60b links chromatin opening to ATM activation}

We next analyzed whether Baf60b mediates chromatin opening and ATM activation. To that end, we first determined whether Baf60b is in the SWI/SNF complex. Immunoprecipitation assays showed that HA-tagged Baf60b interacted with the SWI/SNF complex members, including Brg1, a core ATPase of the SWI/SNF complex (Supplementary information, Figure S10A); but Baf60a and Baf60c were excluded from the complex containing Baf60b (Supplementary information, Figure S10B). Mass spectrometry analysis supported these findings, indicating that the complex is stable during hepatic conversion (Supplementary information, Figure S10 C and Table S10). ChIP assays also showed that Baf60b, Brg1 and Baf170 bound to the open chromatin regions of a panel 

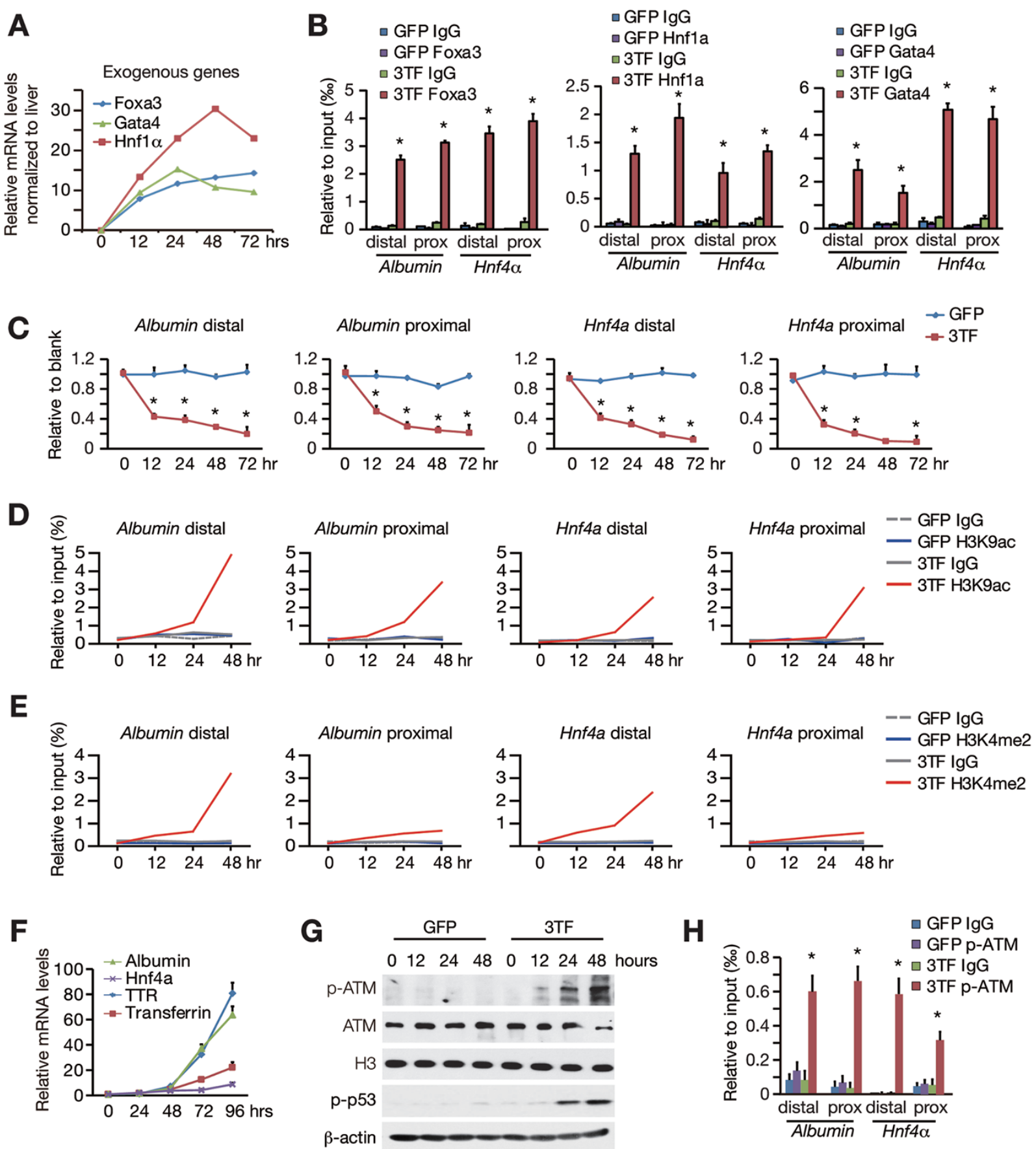

Figure 2 Early molecular events during hepatic conversion. (A) Expression level of 3TF was measured by qRT-PCR at different time points after transduction. (B) Binding of 3TF to regulatory regions of the Albumin and Hnf4 $\alpha$ genes was analyzed by the ChIP assay. Distal and proximal binding sites were determined in Supplementary information, Figure S6. Data represent two independent experiments. (C) Chromatin opening at the Albumin and Hnf4 $\alpha$ genes was measured by the micrococcal nuclease (MNase) digestion assay at different time points after 3TF transduction. Data represent two independent experiments. (D, E) Activated histone marks of H3K9ac (D) and H3K4me2 (E) on the Albumin and Hnf4a genes were examined by the ChIP-qPCR assay at different time points after 3TF transduction. (F) 3TF-induced hepatic gene expression was measured by qRT-PCR at different time points compared to TTFs. (G) p-ATM and p-p53 (Ser15) levels were analyzed by western blot analyses at different time points. (H) Binding of p-ATM to regulatory regions of the Albumin and Hnf4 $\alpha$ genes was analyzed by the ChIP-qPCR assay at $48 \mathrm{~h}$ after 3 TF transduction. Data represent two independent experiments. Error bars indicate SD. * $P<$ 0.05 , Student's $t$-test. 

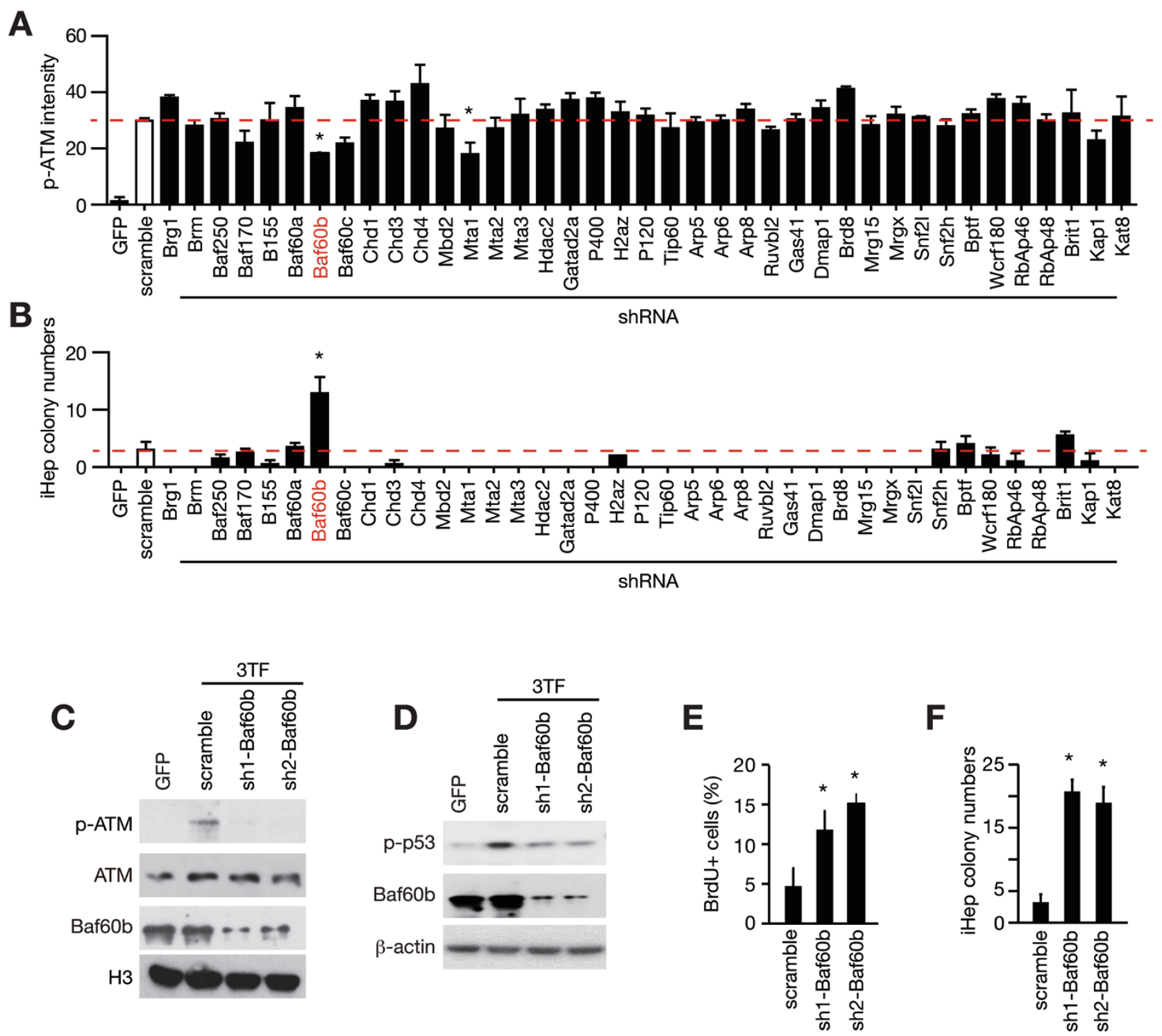

Figure 3 Baf60b mediates 3TF-induced ATM activation. (A) The hepatic conversion was induced in wild-type (WT) TTFs transfected with shRNAs against the indicated genes. Forty-eight hours after 3TF transduction, p-ATM levels were imaged by the Operetta system and quantified by Harmony software. $n=2$ independent experiments, and 350-500 nuclei were analyzed for each shRNA in both experiments. (B) Numbers of iHep colonies were quantified 8 days after 3TF transduction. Data represent two independent experiments. (C-F) The role of Baf60b in ATM activation and iHep formation was confirmed by shRNAs targeting Baf60b CDS (sh1-Baf60b) and 3'UTR (sh2-Baf60b). (C, D) p-ATM and p-p53 (Ser15) levels were analyzed by the western blot assay. (E) Cell proliferation was determined by BrdU incorporation and staining. $n=5$ fields for each group. (F) iHep colony numbers were quantified 8 days after 3TF transduction. $n=4$ independent experiments for each group. Data represent two independent experiments. Error bars indicate SD. * $P<0.05$. Student's $t$-test.

of hepatic gene loci (Figure 4A and Supplementary information, Figure S11A-S11C and Tables S3-S4). Interestingly, Brg1 silencing caused reduced chromatin opening and $\mathrm{H} 3 \mathrm{~K} 9 \mathrm{ac}$ level in hepatic genes (Supplementary information, Figure S12A-S12B and Table S5), whereas Baf60b depletion did not affect these changes (Figure
4B, 4C and Supplementary information, Figure S12C, S12D and Table S5). Given that the levels of Brg1-bound Baf60a and Baf60c were increased in Baf60b-deficient cells (Supplementary information, Figure S13A) and silencing all Baf60 members led to impaired chromatin opening at a panel of hepatic gene loci (Supplementa- 

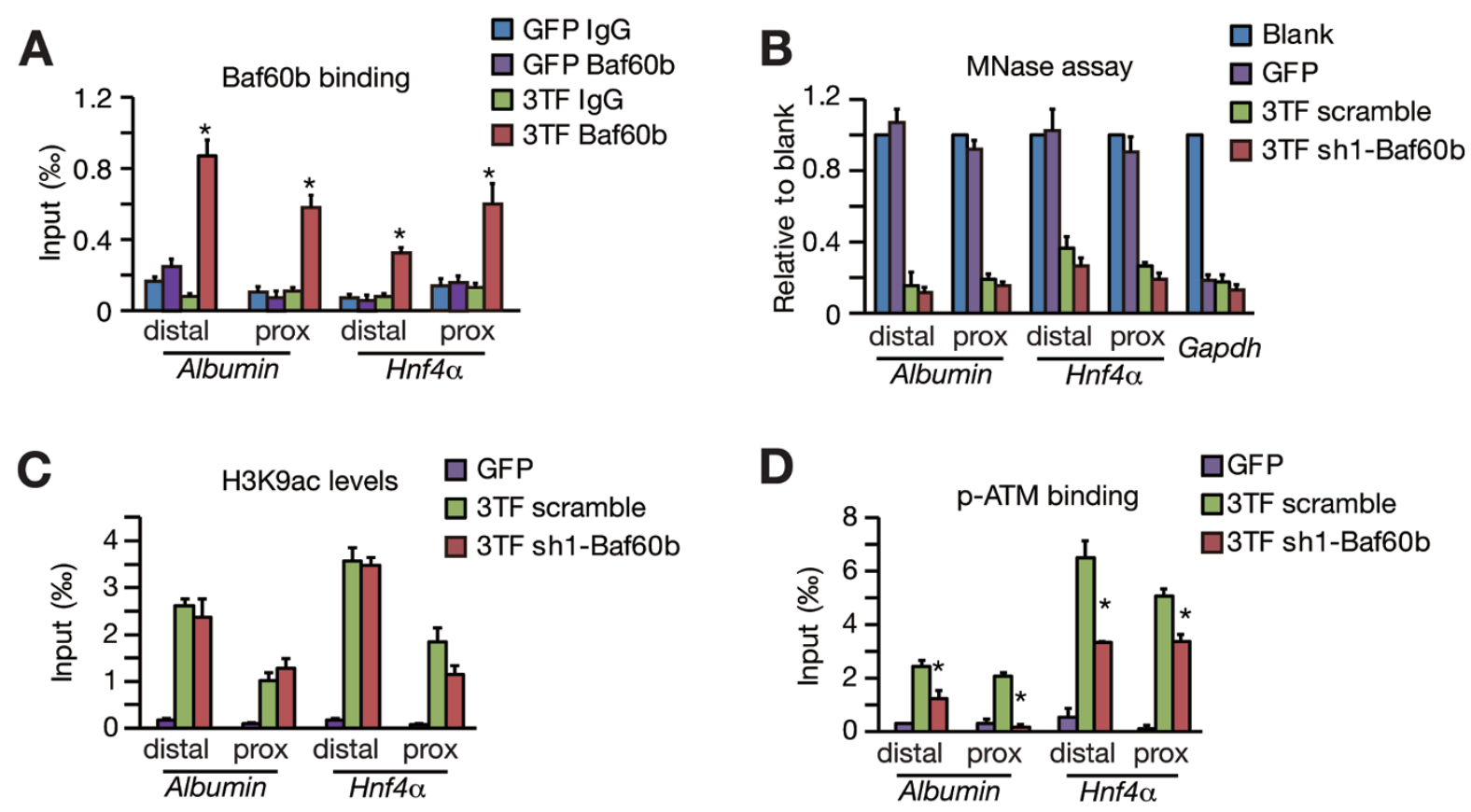

Figure 4 Baf60b links chromatin opening and ATM activation. (A) The binding of Baf60b at the regulatory regions of the Albumin and $H n f 4 \alpha$ genes was determined by the ChIP-qPCR assay. (B) Chromatin opening at the Albumin and Hnf4 $\alpha$ genes was measured by the micrococcal nuclease (MNase) assay in Baf60b-knockdown (sh1-Baf60b) TTFs and scramble controls after 3TF transduction. (C) H3K9ac on the Albumin and Hnf4 $\alpha$ genes was examined in Baf60b-knockdown (sh1-Baf60b) TTFs and scramble controls by the ChIP-qPCR assay. (D) The binding of p-ATM to the Albumin and Hnf4 $\alpha$ genes was analyzed by the ChIP-qPCR assay. sh1-Baf60b-mediated Baf60b silencing attenuated the p-ATM binding. Data represent two independent experiments. Error bars indicate SD. ${ }^{*} P<0.05$. Student's $t$-test.

ry information, Figure S13B and Table S6), it is likely that Baf60a or Baf60c replaced Baf60b functions in Baf60b-deficient cells. Together, these results show that Baf60b is in the SWI/SNF complex and is located at open chromatin regions. A kinetic analysis of the Baf60b and p-ATM binding revealed that Baf60b and p-ATM were sequentially recruited to open chromatin regions of hepatic genes (Supplementary information, Figure S14A and Table S7), suggesting that chromatin recruitment of these two proteins might be associated. Importantly, we found that Baf60b knockdown significantly reduced the binding of p-ATM to open chromatin regions of hepatic genes (Figure 4D and Supplementary information, Figure S14B and Table S8). These data indicate that Baf60b is in the SWI/SNF complex and mediates ATM recruitment to the open chromatin regions at a panel of hepatic gene loci.

Baf60b facilitates the recruitment of ATM to the SWI/ SNF complex

Because the SWI/SNF complex contains no kinase [34, 35 ] and chromatin localization of ATM has an important role in its activation [25], we characterized whether
Baf60b mediates ATM recruitment to open chromatin through the SWI/SNF complex and therefore facilitates its activation. Baf60b was in the SWI/SNF complex as shown by immunoprecipitation assay during hepatic conversion (Figure 5A). Notably, 3TF transduction triggered an interaction between Baf60b and ATM (Figure 5A). We further confirmed the interaction of endogenous Baf60b with ATM and the SWI/SNF complex using monoclonal antibodies specific to Baf60b (Figure 5B). In addition, reverse co-immunoprecipitation using antibodies against $\mathrm{Brg} 1$ confirmed that $3 \mathrm{TF}$ transduction triggered a remarkable interaction between Brg1 and ATM (Figure 5C). Moreover, Baf60b knockdown depleted interactions between Brg1 and ATM (Figure 5D). The 3TF-triggered interaction of Baf60b with the SWI/SNF complex and ATM was also validated by mass spectrometry (Supplementary information, Figure S10C and Table S10). In line with ATM recruitment to the SWI/SNF complex, p-ATM was detected in the Baf60b-precipitated complex (Figure 5A-5C). Furthermore, reduced interaction between ATM and Brg1 was correlated with decreased p-ATM in Baf60b-knockdown cells (Figure 5D). In addition, p53 and phosphorylated p53, as a substrate of 

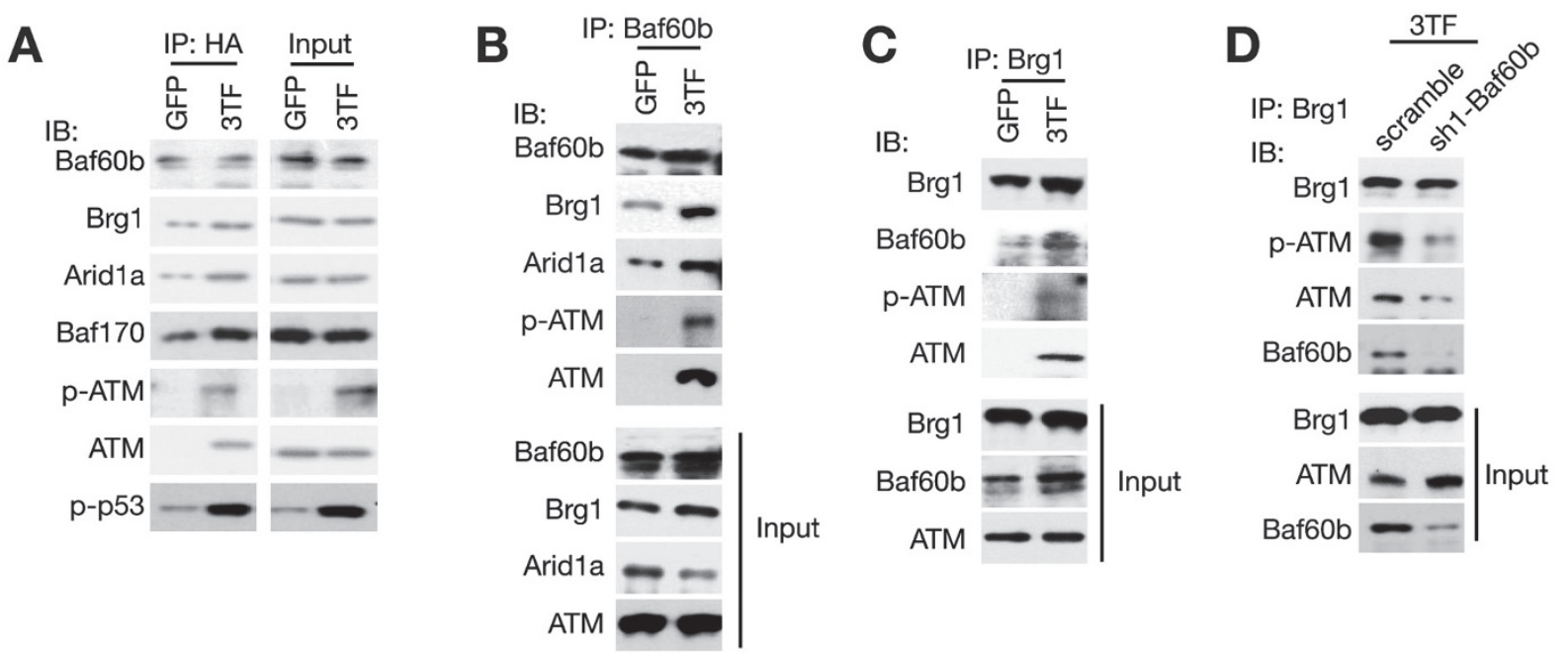

Figure 5 Baf60b facilitates ATM recruitment to the SWI/SNF complex and ATM activation. (A) Analyses of the interaction among Baf60b, the SWI/SNF complex, ATM, p-ATM and p-p53. The hepatic conversion was induced in TTFs transduced with HA-tagged Baf60b. Cell lysates were immunoprecipitated with an HA antibody or control IgG followed by immunoblot (IB) assays with antibodies against the indicated proteins. (B, C) 3TF-transduced TTF lysates were immunoprecipitated with antibodies against endogenous Baf60b (B) or against endogenous Brg1 (C) followed by IB analyses with antibodies against indicated proteins. (D) Baf60b was silenced by sh1-Baf60b in TTFs. Forty-eight hours after 3TF transduction, lysates of TTFs were immunoprecipitated with Brg1 antibody followed by IB analysis with ATM and p-ATM antibodies.

p-ATM, were detected in the Baf60b-precipitated complex (Figure 5A and Supplementary information, Figure S10C and Table S10), suggesting that ATM recruitment and activation are associated. Given that ATMIN controls ATM activation during hepatic conversion (Supplementary information, Figure S5), we analyzed ATM binding in ATMIN-knockdown cells to further dissect ATM recruitment and activation. ATMIN knockdown decreased the amount of Baf60b-bound p-ATM (Supplementary information, Figure S15A) and the recruitment of p-ATM to open chromatin regions of hepatic genes (Supplementary information, Figure S15B and Table S9). In contrast, the amount of Baf60b-bound ATM was unchanged in ATMIN-knockdown cells (Supplementary information, Figure S15A). These results demonstrate a role of Baf60b in recruiting ATM to the SWI/SNF complex after 3TF transduction.

The nuclear location sequence and SWIB domains are necessary for Baf60b-mediated ATM activation

We next investigated whether Baf60b-mediated ATM recruitment is required for ATM activation. We cloned two Baf60b mutants containing the deletion of either the nuclear location sequence $(\triangle \mathrm{NLS})$ or the conserved SWIB domain $(\triangle \mathrm{SWIB})$ of Baf60b (Supplementary information, Figure S16A). The full-length HA-tagged Baf60b was used as a control. Immunofluorescence staining confirmed that $\triangle \mathrm{NLS}$ localized in the cytoplasm, whereas the $\triangle$ SWIB mutant and the HA-tagged Baf60b control localized in the nucleus (Supplementary information, Figure S16B). Due to the cytoplasmic location, the $\triangle$ NLS mutant showed no interaction with the SWI/SNF complex (Supplementary information, Figure S16C) and was unable to recruit ATM after 3TF transduction (Figure $6 \mathrm{~A})$. The $\triangle$ SWIB mutant localized in the nucleus, but it lost the capability to interact with the SWI/SNF complex (Supplementary information, Figures S10C and S16C) and to recruit ATM as shown by immunoprecipitation and mass spectrometry (Figure 6A and Supplementary information, Figure S10C and Table S10). To determine their role in ATM activation, these mutants were expressed in Baf60b-silenced cells in which the endogenous 3'UTR of Baf60b was targeted by sh2-Baf60b. It was notable that these two mutants caused remarkably reduced p-ATM levels compared to the full-length Baf60b control (Figure 6B). As a consequence, both $\triangle \mathrm{NLS}$ and $\triangle \mathrm{SWIB}$ mutants decreased p53 phosphorylation level (Figure 6C) and improved the formation of hepatic colonies (Figure 6D). These results showed that ATM recruitment to the SWI/ SNF complex facilitates its full activation during lineage conversion.

\section{Discussion}

In this study, we identified a molecular basis for the 
A

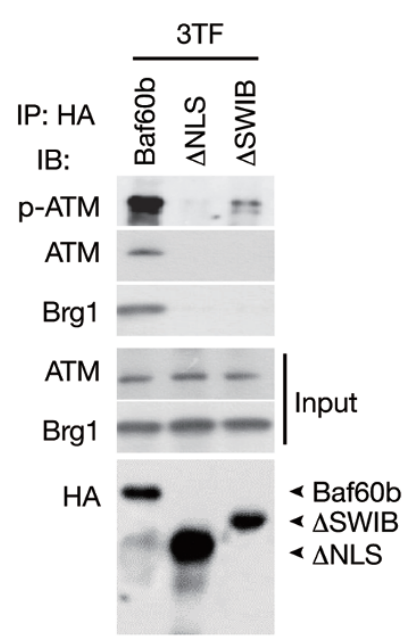

B

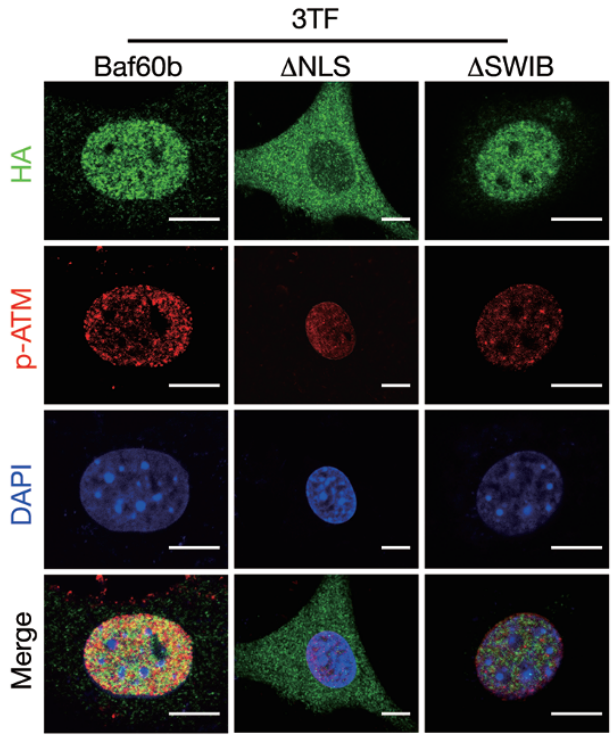

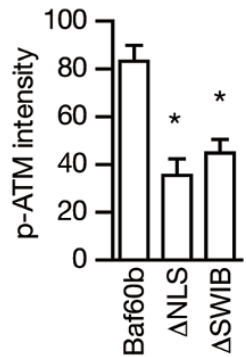

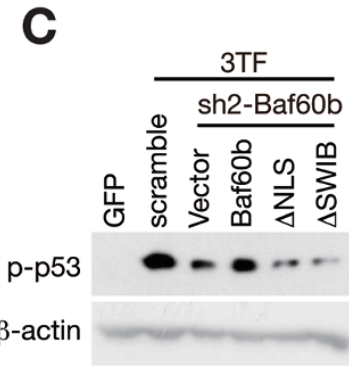

D

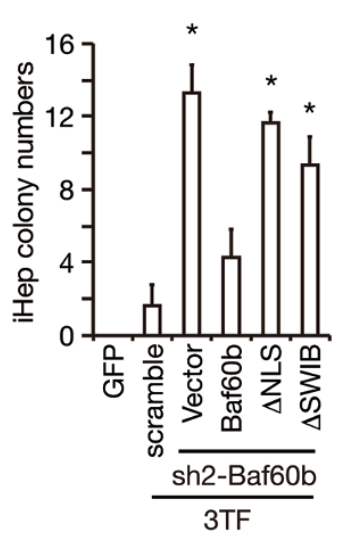

E
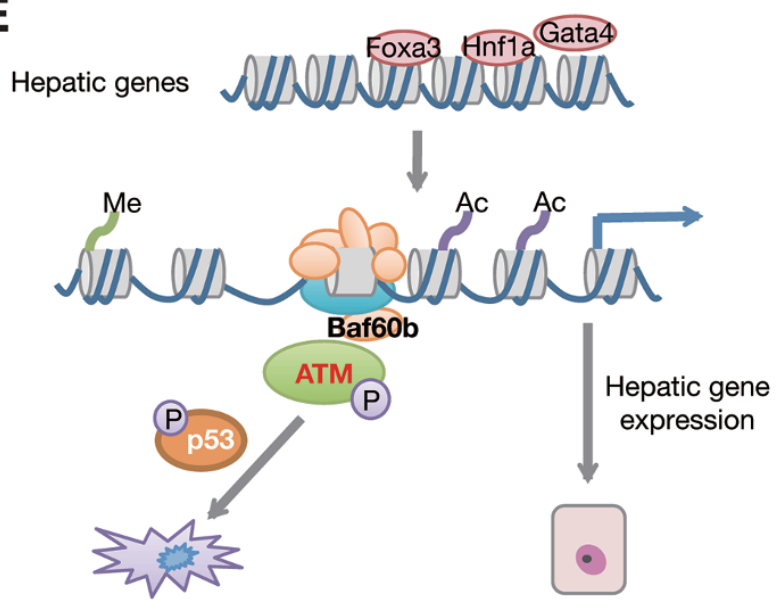

iHep cells

Figure 6 The NLS and SWIB domains are necessary for Baf60b-dependent ATM activation. (A) HA-tagged Baf60b and two Baf60b mutants ( $\triangle$ NLS and $\triangle S$ WIB) were individually transduced into TTFs. Interactions among Baf60b mutants, Brg1, ATM and $\mathrm{p}$-ATM were determined. (B-D) $\triangle \mathrm{NLS}, \triangle \mathrm{SWIB}$ and HA-tagged Baf60b were transduced into Baf60b-silenced TTFs in which the endogenous 3'UTR of Baf60b mRNA was targeted by sh2-Baf60b. 3TF-induced p-ATM was analyzed by immunofluorescence staining and quantified by LAS AF Lite; $n=6$ cells were analyzed for each group (B). p-p53 (Ser15) protein level was determined by the western blot assay $(\mathbf{C})$ and iHep colony numbers were quantified 8 days after iHep induction; $n=4$ independent experiments (D). (E) A schematic model showing that a repressive mechanism involving Baf60b-mediated ATM activation senses chromatin opening and limits hepatic conversion. Error bars indicate SD. ${ }^{*} P<0.05$. Student's $t$-test.

barrier against lineage conversion using $3 \mathrm{TF}$-induced iHep cells as an experimental system. In line with studies on other lineage conversions [36-39], the binding of 3TF to the compact chromatin of lineage-specific genes initiates the hepatic lineage conversion. Transduction of $3 \mathrm{TF}$ triggers the chromatin remodeling complex to open chromatin of hepatocyte-specific genes and to facilitate their expression (Figure 6E). Importantly, we found that $3 \mathrm{TF}$ induce chromatin opening and activate, in parallel, the ATM-p53 pathway, which acts as a negative regulatory loop halting hepatic conversion (Figure 6E). Mechanistically, Baf60b, a component of the SWI/SNF complex, binds to open chromatin regions of hepatic genes and facilitates ATMIN-dependent ATM activation by recruiting ATM to the SWI/SNF complex. Together, we show that ATM activation blocks hepatic conversion by sensing 
chromatin opening at a panel of hepatic gene loci (Figure $6 \mathrm{E}$ ), suggesting that an inappropriately high level of newly opened chromatin regions impedes cell identity conversion by triggering the activation of a "chromatin remodeling checkpoint". Our findings suggest a paradigm of how a differentiated cell resists dramatic cell identity changes.

It is remarkable that the ATM-p53 pathway limits lineage conversion in three seemingly unrelated scenarios, i.e., oncogenic transformation, iPS cell generation and hepatic conversion [17-21, 40]. It remains to be identified whether it is simply a coincidence or whether there are common fundamental mechanisms restricting the plasticity of cell identity. Given that both oncogenic transformation and iPS cell generation are associated with epigenetic remodeling [41-43], it would be worth testing whether in these two processes the ATM-p53 pathway senses chromatin opening and blocks cell identity conversion. Indeed, our preliminary data supported that BAf60b may also be involved in ATM activation during iPS cell induction, although to a lower degree (Supplementary information, Figure S17 and Table S11). It is possible that chromatin remodelers play lineage-specific roles in various cell identity conversion systems.

As a sensor of DNA damage, ATM is activated in response to DNA double-strand break. Activated ATM transduces the signal to DNA damage repair proteins and promotes p53 phosphorylation and stability to maintain the DNA integrity and prevent tumorigenesis [44]. Interestingly, ATM is also activated by chloroquine, hypotonic stress and chromatin-modifying drugs without DNA damage [45], suggesting that ATM activation could result from changes in chromatin conformation. Transcription factor-induced cell lineage conversion is a specific process during which large-scale chromatin remodeling occurs. Our results confirmed that ATM was activated and recruited to $3 \mathrm{TF}$-induced chromatin opening at a panel of hepatic genes. It is interesting to postulate that ATM might be a guard protein that senses the changes in chromatin conformation and helps to maintain the specific identity of a given cell.

We found that Baf60b, a member of the SWI/SNF chromatin remodeling complex, was the key for ATM to sense the chromatin opening. Interestingly, Baf60b appeared not to be essential for chromatin opening. One possible explanation is that after Baf60b depletion, Ba$\mathrm{f} 60 \mathrm{a}$ and Baf60c replaced Baf60b function in opening target chromatin. However, Baf60a and Baf60c could not replace Baf60b in ATM recruitment. shRNA screening data showed that there are low or no compensatory functions between $\mathrm{Baf} 60 \mathrm{a} / \mathrm{b} / \mathrm{c}$ in iHep reprogramming. Specifically, Baf60a knockdown displayed no apparent effect on iHep formation, whereas Baf60c knockdown blocked iHep reprogramming (Figure 3B), suggesting limited ability of Baf60 subunits to compensate for each other in iHep formation. These data also suggested a possible role of Baf60c in mediating chromatin opening. There might be a switch between Baf60 members during chromatin opening and ATM activation. Nevertheless, given that iHep formation is a long-time procedure, the contribution of individual Baf60 members at late stage of iHep induction needs further characterization.

Our results also partially explained why in vitro hepatic conversion is a low-efficiency process. By silencing Baf60b or inactivating the ATM-p53 pathway, the hepatic conversion was allowed to occur at significantly high efficiency. Specifically, Baf60b inactivation would be superior to the inhibition of the p53 pathway by keeping the DNA damage response intact. A careful investigation of the response to epigenetic remodeling might also improve efficiency and quality of other lineage conversions.

\section{Materials and Methods}

\section{Mice and isolation of TTFs}

All mice used in this study were maintained under specific pathogen-free conditions at the animal facility of Institute of Biochemistry and Cell Biology, Chinese Academy of Sciences. TTFs were isolated from 4-week-old male C57/B6 mice or genetically modified mice. $\sim 2 \mathrm{~cm}$ length of tail tip was cut and washed with $70 \%$ ethanol and PBS. After dermis was peeled away, the remaining part was placed into a $60-\mathrm{mm}$ collagen-I-coated dish in $5 \mathrm{ml}$ DMEM (Hyclone) containing 10\% FBS (Gibco). After 5 days incubation, fibroblasts that migrated out were trypsinized and expanded to a $10-\mathrm{cm}$ collagen-I-coated dish. We used TTFs at passage 3 for iHep cell induction. All mouse experiments were approved by the Institutional Animal Care and Use Committee (IACUC) of the Institute of Biochemistry and Cell Biology and performed in accordance with the committee's guidelines.

\section{iHep cell induction}

iHep cells were generated as previously described [12]. Briefly, $10^{5}$ TTFs at passage 3 were seeded on a collagen-I-coated $6 \mathrm{~cm}$ dish and transfected with lentiviruses carrying 3TF. Cells were collected for further analyzing at different time points according to specific experiment. We quantified the colonies at day 8 or later after iHep induction. At this time point, epithelial cell colonies were visible and could be quantified. In previous study, we have demonstrated that these individual iHep colonies were similar to each other [12]. Some of the colonies were additionally confirmed as iHep cells by immunofluorescence staining for Albumin and E-cadherin.

\section{Molecular cloning and lentivirus production}

The constructions of $3 \mathrm{TF}$ were described previously [12]. Baf60b, $\triangle$ NLS and $\triangle$ SWIB cDNA with HA-Tag at N-terminus were cloned into the modified pWPI plasmid (Addgene). The NLS domain distributes from 122 aa to 207 aa of Baf60b protein as indicated by our results. The SWIB domain (307 aa to 385 aa) was 
determined according to the previous report [46]. For shRNA-mediated gene silencing, DNA oligonucleotides targeting each gene were inserted into the AgeI and EcoRI restriction enzyme sites of the pLKO.1 plasmid, respectively. DNA sequences were mainly obtained from Sigma MISSION shRNA library. The oligonucleotide sequences are provided in Supplementary information, Table S12. Constructed pWPI or pLKO.1 plasmids were introduced into 293FT cells together with packaging plasmid psPAX2 (Addgene) and envelope plasmid pMD2.G (Addgene). After $48 \mathrm{~h}$ of incubation, lentiviruses were collected and stored at $-80{ }^{\circ} \mathrm{C}$.

\section{Immunofluorescence staining assays}

Cells were fixed with $4 \%$ paraformaldehyde for $15 \mathrm{~min}$ at room temperature, and then blocked with PBS containing $0.25 \%$ Triton $\mathrm{X}-100$ (Sigma) and 3\% BSA (Sigma) for $1 \mathrm{~h}$ at room temperature. The cells were then incubated with primary antibodies at $4{ }^{\circ} \mathrm{C}$ overnight, washed with PBS, and incubated with appropriate fluorescence-conjugated secondary antibody for $1 \mathrm{~h}$ at room temperature in the dark. Nuclei were stained with DAPI (Sigma). Primary and secondary antibodies were diluted in PBS containing 3\% BSA. Images were captured at room temperature using an Olympus microscope (IX71) or a confocal microscope (TCS SP5; Leica) with a $63 \times$ oil objective. The p-ATM intensity was quantified by software LAS AF Lite (Leica). Antibodies used for immunofluorescence are as follows: rabbit anti-E-cadherin (Cell Signaling, 1:200), mouse anti-albumin (R\&D, 1:200), mouse anti-p-ATM (Ser1981) (Thermo, 1:200), rabbit anti-HA-Tag (Cell Signaling, 1:200), rat anti-RPA32 (Cell Signaling, 1:200), rabbit anti-53BP1 (Novus, 1:200), goat anti-Nanog (R\&D, 1:100), mouse anti-SSEA1 (R\&D, 1:100), Cy3-conjugated donkey anti-mouse/rabbit/rat/goat IgG (Jackson Laboratories, 1:1 000), Cy5-conjugated goat anti-rabbit/ mouse IgG (Jackson Laboratories, 1:1 000).

\section{5-bromodeoxyuridine (BrdU) incorporation assay}

Cells were incubated with BrdU (BrdU Labeling Reagent, Ready-to-use, Invitrogen, 1:100 diluted in culture medium) for 6 $\mathrm{h}$ at $37^{\circ} \mathrm{C}$. Cells were washed with PBS and fixed in acid ethanol $\left(90 \%\right.$ ethanol, $5 \%$ acetic acid, $5 \% \mathrm{H}_{2} \mathrm{O}$ ) for $30 \mathrm{~min}$ at room temperature, followed by incubation in $2 \mathrm{M} \mathrm{HCl}$ for $20 \mathrm{~min}$ at room temperature. $0.1 \mathrm{M}$ sodium borate $(\mathrm{pH} 8.5)$ was added to the cells and incubated for another $2 \mathrm{~min}$. Cells were washed with PBS and incubated with BrdU antibody (Cell Signaling, 1:1 400) in 3\% BSA-PBS at $4{ }^{\circ} \mathrm{C}$ overnight. After washed with PBS, the cells were incubated with $\mathrm{Cy} 3$-conjugated donkey anti-mouse IgG. Nuclei were stained with DAPI (Sigma). The BrdU-positive cells were counted using an Olympus microscope (IX71).

\section{SA- $\beta$-Gal staining}

SA- $\beta$-Gal staining was performed according to the manufacturer's instruction (Beyotime). Briefly, $5 \times 10^{4} \mathrm{TTF}$ cells were placed in a 6 -well plate and transduced with $3 \mathrm{TF}$ or empty vector and cultured for 6 days. After being washed with PBS, cells were fixed and then incubated with staining working solution overnight at 37 ${ }^{\circ} \mathrm{C}$. The senescent cells were counted under an Olympus microscope (IX71).

\section{Flow cytometry}

For apoptosis analysis, cells were collected at day 6 after $3 \mathrm{TF}$ or empty vector transfection. Apoptotic cells were detected by An- nexin V Apoptosis Detection Kit APC (eBioscience) and analyzed by FACS Calibur. The production of reactive oxygen species (ROS) was detected $48 \mathrm{~h}$ after $3 \mathrm{TF}$ transduction using CellROX ${ }^{\mathrm{TM}}$ Deep Red Reagent (Invitrogen). Cells were incubated with CellROX ${ }^{\mathrm{TM}}$ Deep Red Reagent at a final concentration of $5 \mu \mathrm{M}$ for $30 \mathrm{~min}$ at $37{ }^{\circ} \mathrm{C}$. The labeled cells were washed with PBS and analyzed by FACS Calibur (Becton Dickinson).

\section{Comet assay}

The microscope slide was pre-treated with $10 \%$ polylysine and coated with a thin $1 \%$ normal melting point agarose (NMA) gel. Cells were collected at $48 \mathrm{~h}$ after $3 \mathrm{TF}$ transfection, suspended in $1 \%$ low melting point agarose (LMA) and embedded within the NMA gel on the slide. The slide was put in the lysis buffer $(2.5$ $\mathrm{mM} \mathrm{NaCl}, 100 \mathrm{mM}$ EDTA, $10 \mathrm{mM}$ Tris base, 1\% Triton X-100) at $4{ }^{\circ} \mathrm{C}$ for $1 \mathrm{~h}$ to remove the cellular proteins. The DNA was allowed to unwind in the alkaline $\mathrm{pH}$ conditions $(300 \mathrm{mM} \mathrm{NaOH}, 1 \mathrm{mM}$ EDTA) at room temperature for $1.5 \mathrm{~h}$ and then electrophoresed at $25 \mathrm{~V}, 300 \mathrm{~mA}$ for $20 \mathrm{~min}$. The DNA was stained with ethidium bromide. Pictures were taken by an Olympus microscope (BX51). The comet tail length was measured by CASP software.

\section{Micrococcal nuclease (MNase) digestion assay}

The MNase digestion assay was performed as described before with some modifications [47]. $5 \times 10^{5}$ cells were collected and suspended in Buffer A (10 mM HEPES pH7.9, $1.5 \mathrm{mM} \mathrm{MgCl}_{2}$, $10 \mathrm{mM} \mathrm{KCl}, 0.5 \mathrm{mM}$ DTT) supplemented with protease inhibitor cocktail (Sigma) on ice for $15 \mathrm{~min}$. NP40 was then added to $0.5 \%$ concentration followed by vortex for $20 \mathrm{~s}$. The nuclei pellets were collected by centrifuged at $4000 \mathrm{rpm}$ for $5 \mathrm{~min}$. The nuclei was washed with $2.5 \mathrm{ml}$ of MNase digestion buffer $(10 \mathrm{mM}$ Tris- $\mathrm{HCl}$ $\mathrm{pH} 7.4,15 \mathrm{mM} \mathrm{NaCl}, 60 \mathrm{mM} \mathrm{KCl}, 0.15 \mathrm{mM}$ spermine, $0.5 \mathrm{mM}$ spermidine) and centrifuged to collect the pellet. The nuclei was resuspended in $1 \mathrm{ml}$ of MNase digestion buffer containing $1 \mathrm{mM}$ $\mathrm{CaCl}_{2} .100 \mu \mathrm{l}$ of nuclei were transferred to a series of microcentrifuge tubes containing $2 \mathrm{U}$ of MNase (Takara). Samples were incubated at room temperature for $5 \mathrm{~min} .80 \mu \mathrm{l}$ of MNase digestion buffer and $20 \mu \mathrm{l}$ MNase stop buffer (100 mM EDTA, $10 \mathrm{mM}$ EGTA pH7.5) were added to each sample. Proteinase K was added to a final concentration of $0.2 \mathrm{mg} / \mathrm{ml}$. $10 \mu 1$ of $20 \%$ SDS was added. After incubation at $55{ }^{\circ} \mathrm{C}$ for $4 \mathrm{~h}$, DNA was purified using MinElute PCR Purification Kit (Qiagen) and characterized by qPCR. Primer sequences are provided in Supplementary information, Tables S1 and S13.

\section{Chromatin immunoprecipitation}

Cells were washed with PBS and crosslinked with 1\% formaldehyde for $10 \mathrm{~min}$. A final concentration of $0.2 \mathrm{M}$ Glycine was added to quench the crosslinking for $5 \mathrm{~min}$, and then the cells was washed with PBS. Nuclei were isolated and resuspended in lysis buffer (10mM Tris-HCl, pH 8.0, 200mM NaCl, 1mM EDTA, $0.5 \mathrm{mM}$ EGTA, $0.1 \%$ Na-Deoxycholate, $0.5 \%$ N-lauroylsarcosine sodium salt, $0.2 \%$ SDS, protease inhibitor) for sonication, followed by sonication (Covaris S220) to yield DNA fragments around 200-500 bp. The lysate was centrifuged. A certain amount of supernatant was used for input. After adding the incubation buffer to a final concentration of $1 \times$ RIPA ( $1 \%$ Triton X-100, $0.1 \%$ SDS, $0.1 \%$ Na-Deoxycholate, in TE buffer) with $0.4 \mathrm{M} \mathrm{NaCl}$ and protease inhibitor, DNA was co-immunoprecipitated using ChIP- 
grade antibodies against Foxa3, Gata4 and Hnfla (Santa Cruz), H3K9ac and H3K4me2 ChIP-grade antibodies (Abcam), p-ATM (Ser1981) antibody (Thermo), and Baf60b antibody (Abnova) and Brg1(Santa Cruz) and Baf170 (Bethyl laboratories) or control IgG (normal mouse and rabbit IgG from Millippore, normal goat IgG from Santa Cruz) at $4{ }^{\circ} \mathrm{C}$ overnight. The immunoprecipitates were collected by incubating with Dynabeads Protein G (Life Technologies) at $4{ }^{\circ} \mathrm{C}$ for $2 \mathrm{~h}$, and sequentially washed with RIPA buffer $(1 \%$ Triton X-100, $0.1 \%$ SDS, $0.1 \%$ Na- Deoxycholate, in TE) twice, RIPA buffer with $0.3 \mathrm{M} \mathrm{NaCl}$ twice, $\mathrm{LiCl}$ buffer $(0.25 \mathrm{M} \mathrm{LiCl}, 0.5 \%$ NP40, 1\% deoxycholic acid, 1 mM EDTA, 10 mM Tris-HCl pH 8.0) twice, TE buffer (10 mM Tris-HCl pH 8.0, 1 mM EDTA) with $0.2 \%$ Triton X-100 once and TE buffer once. The immunoprecipitates and the input DNA were reverse-crosslinked with $0.2 \mathrm{mg} / \mathrm{ml}$ proteinase $\mathrm{K}$ at $65{ }^{\circ} \mathrm{C}$ for $2 \mathrm{~h}$ and finally eluted from the beads using Elution buffer (Qiagen) and treated with RNase A at $37{ }^{\circ} \mathrm{C}$ for 30 min. DNA fragments and input DNA were purified using MinElute PCR Purification Kit (Qiagen) and the DNA concentration is quantified by Qbit (Life Technologies). The same amount of DNA in each panel of experiment is used for qPCR. Primer sequences are provided in Supplementary information, Tables S1 and S13.

To characterize a panel of hepatic genes in this study using ChIP-qPCR, we tested 34 candidate hepatic genes based on published data [48-50]. Next, we designed PCR primer pairs for 205 sites located in the 34 genes to identify 3TF-binding loci (Supplementary information, Table S1). By ChIP-qPCR assay, we found 81 sites with enriched 3TF-binding in 30 out of the 34 candidate genes (Supplementary information, Table S2). We selected 32 sites on 24 genes for further analyses (Supplementary information, Table S3). The 32 sites were selected by their distant location from each other.

\section{Immunoprecipitation and immunoblot analysis}

For immunoprecipitation assay, TTFs were transfected with $3 \mathrm{TF}$ and respective cDNA clone (HA-Baf60b, HA- $\triangle \mathrm{NLS}$, HA$\triangle \mathrm{SWIB}$ ) in $\mathrm{pWPI}$ vector (modified from Addgene). Cells were suspended in Buffer A (10 mM HEPES pH7.9, $1.5 \mathrm{mM} \mathrm{MgCl}_{2}$, $10 \mathrm{mM} \mathrm{KCl}, 0.5 \mathrm{mM}$ DTT) supplemented with protease inhibitor cocktail (Sigma) on ice for $15 \mathrm{~min}$. NP40 was then added into a final concentration of $0.5 \%$ followed by vortex for $20 \mathrm{~s}$. The nuclear pellets were collected by centrifugation at $4000 \mathrm{rpm}$ for $5 \mathrm{~min}$. The nuclei were lysed in buffer (50 mM Tris- $\mathrm{HCl} \mathrm{pH} 7.4,150 \mathrm{mM}$ $\mathrm{NaCl}, 1 \mathrm{mM}$ EDTA, $1 \%$ Triton X-100 and 0.1\% NP40) supplemented with protease inhibitor cocktail. Lysates were incubated with appropriate antibody or $\operatorname{IgG}$ at $4{ }^{\circ} \mathrm{C}$ overnight before adding Protein G-conjugated Dynabeads for $2 \mathrm{~h}$. The beads were washed three times with lysis buffer and eluted with SDS loading buffer by boiling for $5 \mathrm{~min}$, and subjected to immunoblot analysis. For immunoblot analysis, the samples were separated by SDS-PAGE, and transferred to a PVDF membrane (Millipore). The membrane was blocked with $5 \%(\mathrm{w} / \mathrm{v})$ reagent-grade nonfat milk (Cell Signaling Technology) and incubated with primary antibodies at $4{ }^{\circ} \mathrm{C}$ overnight followed by secondary antibody incubation. The protein bands were visualized using Clarity ${ }^{\mathrm{TM}}$ Western ECL substrate (Bio-Rad).

\section{Mass spectrometry and data analysis}

The Co-IP samples were separated on an $8 \%$ SDS-PAGE, and then stained with Coomassie Blue. The gel lanes were cut into eight bands per sample and in-gel trypsin digestion was performed strictly following a reported protocol [50]. Liquid chromatography separation was performed on a Proxeon Easy-nanoLC 1000 system (ThermoFisher Scientific). The LC column (15-cm length, 75$\mu \mathrm{m}$ inner diameter) was packed in-house with ReproSil-Pur C18AQ $3 \mu \mathrm{m}$ resin (Dr Maisch $\mathrm{GmbH}$ ) in $100 \%$ methanol. For high mass accuracy data acquisition, the Orbitrap Fusion Tribrid mass spectrometer (ThermoFisher Scientific) was used and equipped with a nano-electrospray ion source (Proxeon Biosystems, now ThermoFisher Scientific). The peptides were dissolved in $0.1 \%$ FA, loaded onto the column and separated with a linear gradient of $4 \%-30 \%$ buffer $\mathrm{B}$ (ACN with $0.1 \%$ formic acid) at a flow rate of $300 \mathrm{~nL} / \mathrm{min}$ over $43 \mathrm{~min}$. The Fusion was operated using 'highlow' mode, in which during a maximum 3 s cycle time, the most abundant multiply charged parent ions were selected with high resolution $(120000,200 \mathrm{~m} / \mathrm{z})$ from the full scan $(300-1500 \mathrm{~m} / \mathrm{z})$ for HCD fragmentation. For MS/MS spectra, ion trap detector was used by a "rapid" scan rate with a target value of 2e4 (AGC control enabled) and an isolation window of $2 \mathrm{~m} / \mathrm{z}$. The normalized collision energy was set to $33 \%$.

Raw data files were processed and analyzed using the MaxQuant software (Version. 1.5.2.8). The MS/MS spectra were searched against the mouse Uniprot protein database (201405) using the Andromeda search engine. The spectra were searched with a mass tolerance of $6 \mathrm{ppm}$ in MS mode, 0.5 Da in HCD MS/ MS mode, strict trypsin specificity, and allowing up to 2 missed cleavage sites. The minimum required peptide length was 6 amino acids. Cysteine carbamidomethylation was set as a fixed modification, whereas $\mathrm{N}$-acetyl protein, oxidized methionine and acetylation of lysine were searched as variable modifications. The peptide and protein false discovery rate (FDR) was fixed at no more than 0.01 . The LFQ intensity was used to quantify the abundance of proteins identified in each sample after normalization by total protein intensity and the intensity of Baf60b (Smarcd2).

\section{shRNA screening of chromatin remodelers for ATM activa- tion}

TTFs were transduced with lentivirus carrying shRNA targeting specific chromatin remodelers, and re-seeded to 24-well plate at the density of 15000 cells per well followed by transfection with 3TF lentiviruses. p-ATM activation was analyzed by immunofluorescence quantification $48 \mathrm{~h}$ after $3 \mathrm{TF}$ transfection. Plate scan and image collection were performed on the Operetta HTS imaging system (PerkinElmer) at $20 \times$ magnification with 15 fields of view, Images were then analyzed with Harmony (PerkinElmer). iHep cell colony numbers were counted at day 8 after $3 \mathrm{TF}$ transduction.

\section{Glycogen storage, DiI-ac-LDL uptake, CYP metabolism as- say, Albumin ELISA and tumor generation assays}

WT TTFs were infected with lenti-virus carrying shNT, sh1Baf60b respectively for $48 \mathrm{~h}$. Cells were transduced with $3 \mathrm{TF}$ for iHep cell generation. The hepatic function assays were done at day 21 after $3 \mathrm{TF}$ transfection. Glycogen storage was quantified by colorimetric measurement by Glycogen Assay Kit (Abnova). iHep cells were stained by DiI-ac-LDL (Invitrogen) following the manufacturer's instructions. For the measurement of CYP enzyme induction, iHep cells were cultured in Block's medium supplemented with 3-methylcholanthrene $(25 \mu \mathrm{M})$ and sodium phenobarbital $(1 \mathrm{mM})$ for $48 \mathrm{~h}$. Cells were incubated with substrate in 
$200 \mu \mathrm{l}$ incubation medium at $100 \mu \mathrm{M}$ for $3 \mathrm{~h}$ at $37^{\circ} \mathrm{C}$. To stop the reaction, $800 \mu \mathrm{l}$ of cold methanol was added and centrifuged. The supernatants were collected for measurement of metabolized compounds by LC-MS/MS (Agilent 1200 HPLC and ABI 4000 mass-spectrometer). Total cell protein amount was used to normalize the data. The amount of secreted Albumin was determined by the mouse Albumin ELISA kit (Bethyl Laboratory) according to the manufacturer's instructions.

For the tumor generation assay, human hepatoma cell line SNU398 was used as positive control. Three nude mice were injected with $3 \times 10^{6}$ iHep cells in the left subcutaneous flank and 3 $\times 10^{6} \mathrm{SNU} 398$ cells in the right subcutaneous flank. Tumor formation was examined 8 weeks after injection.

\section{iPS cell generation and characterization}

WT TTFs were infected with lentiviruses carrying shNT, sh1Baf60b and sh2-Baf60b respectively for 48 hours. Cells were reseeded and transfected with retrovirus carrying Oct4, Sox2, Klf4, and c-Myc for iPS cell induction [10]. The iPS cells were maintained on mitomycin C-treated MEF in DMEM/F12 culture medium supplemented with $20 \%$ KnockOut serum replacement, $0.1 \mathrm{mM}$ non-essential amino acids, $1 \mathrm{mM}$ L-glutamine, $0.1 \mathrm{mM}$ $\beta$-mercaptoethanol and LIF. iPS colonies were picked for expansion around 14 days after transduction. iPS cells were characterized for alkaline phosphatase activity following the procedures in Alkaline Phosphatase kits (Sigma-Aldrich). AP positive colonies were counted to quantify the iPS induction efficiency at day 14 after transduction. The induction of Nanog and SSEA1 was measured by immunofluorescence staining. Pluripotent genes induction was determined by qRT-PCR. Primer sequences are provided in Supplementary information, Table S14.

\section{CRISPR/Cas9-mediated Baf60b knockout}

We purchased $\mathrm{Baf6Ob}^{+-}\left(\mathrm{Smarcd} 2^{\mathrm{tm} 1.1 \text { (KOMP)Vlcg }}\right)$ mice from Knockout Mouse Project (KOMP) Repository located at University of California Davis. We were not able to obtain $\mathrm{Baf}_{6} \mathrm{Ob}^{-/-}$mice by breeding Baf60 $\mathrm{b}^{+/-}$mice. Baf60b-specific sgRNA oligos were designed by the CRISPR website (http://crispr.mit.edu/) targeting sequences at exon 4 (5'-CCTCTCGAAAGCTAAAAGATCC-3'). The oligos were cloned into the lentiCRISPR V2 plasmid [52]. For efficient Baf60b knockout, we used $\mathrm{Baf}_{60 \mathrm{O}}{ }^{+/-}$TTF cells as starting cells with serial lentiCRISPR V2-sgBaf60b virus transfection. We used puromycin to select the transfected cells. We used mixed Baf60b-knockout cells in the experiments. Genomic DNA was extracted from the mixed cells and the Baf60b knockout efficiency was analyzed by amplicon sequencing. Out of the 15 sequenced $B a f 60 b$ genome PCR fragments, 14 showed nonsense mutations in the targeted region of the $B a f 60 b$ gene (data not shown).

\section{Statistics}

All data are presented as the mean + SD. For statistic evaluation, unpaired Student's $t$-test was applied for calculating statistical probability in this study. The differences were considered significant when one-tailed $P<0.05$.

\section{Acknowledgments}

We are grateful to Dr Haikun Liu (DKFZ, Germany), Dr Ping $\mathrm{Hu}$ (SIBS, China), Dr Xiaochun Yu (Beckman Research Institute,
City of Hope, USA) and Dr Xin Wang (Inner Mongolia University, China) for critical reading of the manuscript. We thank Dr Fajian Hou (SIBS, China) for the help on protein immunoprecipitation assays. We appreciate the suggestions from Dr Fulai Jin (Case Western Reserve University, USA) and Dr Feng Liu (UCSD, USA) on ChIP assay. This study is supported by the National Natural Science Foundation of China (31225016, 91319307 and 81471948), the Ministry of Science and Technology of China (2013CB967103 and 2012CB945001), Strategic Priority Research Program of the Chinese Academy of Sciences (XDA01030601) and the Science and Technology Commission of Shanghai Municipality (14XD1404200 and 15JC1400200).

\section{Author Contributions}

SJ and LH conceived the project. SJ performed most experiments with the support from LZ. LZ constructed the shRNA lentiviral vectors and performed the ChIP-PCR assay with the help from Yawei G and SG. Yimeng G conducted iPS reprogramming and characterization. YY designed the sgRNA for Baf60b. XZ analyzed the ChIP-seq data. SJ and RL conducted mass spectrum and data processing under the conduction of RZ and LL. JC was responsible for the mouse maintenance. $\mathrm{CH}$ and $\mathrm{GW}$ were involved in experimental design, data analyses and manuscript writing. SJ and $\mathrm{LH}$ wrote the manuscript.

\section{Competing Financial Interests}

The authors declare no competing financial interests.

\section{References}

1 Tetteh PW, Farin HF, Clevers H. Plasticity within stem cell hierarchies in mammalian epithelia. Trends Cell Biol 2015; 25:100-108.

2 Sanchez AA, Yamanaka S. Rethinking differentiation: stem cells, regeneration, and plasticity. Cell 2014; 157:110-119.

3 Kami D, Gojo S. Tuning cell fate: from insights to vertebrate regeneration. Organogenesis 2014; 10:231-240.

4 Papp B, Plath K. Reprogramming to pluripotency: stepwise resetting of the epigenetic landscape. Cell Res 2011; 21:486501.

5 Holmberg J, Perlmann T. Maintaining differentiated cellular identity. Nat Rev Genet 2012; 13:429-439.

6 Vierbuchen T, Wernig M. Molecular roadblocks for cellular reprogramming. Mol Cell 2012; 47:827-838.

7 Buganim Y, Faddah DA, Jaenisch R. Mechanisms and models of somatic cell reprogramming. Nat Rev Genet 2013; 14:427439.

8 Xu J, Du Y, Deng H. Direct lineage reprogramming: strategies, mechanisms, and applications. Cell Stem Cell 2015; 16:119-134.

9 Ieda M, Fu JD, Delgado-Olguin P, et al. Direct reprogramming of fibroblasts into functional cardiomyocytes by defined factors. Cell 2010; 142:375-386.

10 Takahashi K, Yamanaka S. Induction of pluripotent stem cells from mouse embryonic and adult fibroblast cultures by defined factors. Cell 2006; 126:663-676.

11 Vierbuchen T, Ostermeier A, Pang ZP, Kokubu Y, Sudhof TC, Wernig M. Direct conversion of fibroblasts to functional neurons by defined factors. Nature 2010; 463:1035-1041. 
12 Huang $\mathrm{P}, \mathrm{He} \mathrm{Z}$, Ji S, et al. Induction of functional hepatocyte-like cells from mouse fibroblasts by defined factors. $\mathrm{Na}$ ture 2011; 475:386-389.

13 Huang P, Zhang L, Gao Y, et al. Direct reprogramming of human fibroblasts to functional and expandable hepatocytes. Cell Stem Cell 2014; 14:370-384.

14 Ji S, Zhang L, Hui L. Cell fate conversion: direct induction of hepatocyte-like cells from fibroblasts. J Cell Biochem 2013; 114:256-265.

15 Vousden KH, Lane DP. p53 in health and disease. Nat Rev Mol Cell Biol 2007; 8:275-283.

16 Vazquez A, Bond EE, Levine AJ, Bond GL. The genetics of the p53 pathway, apoptosis and cancer therapy. Nat Rev Drug Discov 2008; 7:979-987.

17 Hong H, Takahashi K, Ichisaka T, et al. Suppression of induced pluripotent stem cell generation by the p53-p21 pathway. Nature 2009; 460:1132-1135.

18 Kawamura T, Suzuki J, Wang YV, et al. Linking the p53 tumour suppressor pathway to somatic cell reprogramming. Nature 2009; 460:1140-1144.

19 Li H, Collado M, Villasante A, et al. The Ink4/Arf locus is a barrier for iPS cell reprogramming. Nature 2009; 460:11361139.

20 Marion RM, Strati K, Li H, et al. A p53-mediated DNA damage response limits reprogramming to ensure iPS cell genomic integrity. Nature 2009; 460:1149-1153.

21 Utikal J, Polo JM, Stadtfeld M, et al. Immortalization eliminates a roadblock during cellular reprogramming into iPS cells. Nature 2009; 460:1145-1148.

22 Evan GI, Wyllie AH, Gilbert CS, et al. Induction of apoptosis in fibroblasts by c-myc protein. Cell 1992; 69:119-128.

23 Lowe SW, Bodis S, McClatchey A, et al. p53 status and the efficacy of cancer therapy in vivo. Science 1994; 266:807810.

24 Stucki M, Clapperton JA, Mohammad D, Yaffe MB, Smerdon SJ, Jackson SP. MDC1 directly binds phosphorylated histone $\mathrm{H} 2 \mathrm{AX}$ to regulate cellular responses to DNA double-strand breaks. Cell 2005; 123:1213-1226.

25 Paull TT. Mechanisms of ATM Activation. Annu Rev Biochem 2015; 84:711-738.

26 Liao W, McNutt MA, Zhu WG. The comet assay: a sensitive method for detecting DNA damage in individual cells. Methods 2009; 48:46-53.

27 Giglia-Mari G, Zotter A, Vermeulen W. DNA damage response. Cold Spring Harb Perspect Biol 2011; 3:a000745.

28 Lee JH, Paull TT. Direct activation of the ATM protein kinase by the Mre11/Rad50/Nbs1 complex. Science 2004; 304:9396.

29 Kanu N, Behrens A. ATMIN defines an NBS1-independent pathway of ATM signalling. EMBO J 2007; 26:2933-2941.

30 Zhang T, Penicud K, Bruhn C, et al. Competition between NBS1 and ATMIN controls ATM signaling pathway choice. Cell Rep 2012; 2:1498-1504.

31 Si-Tayeb K, Lemaigre FP, Duncan SA. Organogenesis and development of the liver. Dev Cell 2010; 18:175-189.

32 Cirillo LA, Lin FR, Cuesta I, Friedman D, Jarnik M, Zaret KS. Opening of compacted chromatin by early developmental transcription factors HNF3 (FoxA) and GATA-4. Mol Cell 2002; 9:279-289.
33 Zaret KS, Watts J, Xu J, Wandzioch E, Smale ST, Sekiya T. Pioneer factors, genetic competence, and inductive signaling: programming liver and pancreas progenitors from the endoderm. Cold Spring Harb Symp Quant Biol 2008; 73:119-126.

34 Hargreaves DC, Crabtree GR. ATP-dependent chromatin remodeling: genetics, genomics and mechanisms. Cell Res 2011; 21:396-420.

35 Swygert SG, Peterson CL. Chromatin dynamics: interplay between remodeling enzymes and histone modifications. Biochim Biophys Acta 2014; 1839:728-736.

36 Polo JM, Anderssen E, Walsh RM, et al. A molecular roadmap of reprogramming somatic cells into iPS cells. Cell 2012; 151:1617-1632.

37 Soufi A, Donahue G, Zaret KS. Facilitators and impediments of the pluripotency reprogramming factors' initial engagement with the genome. Cell 2012; 151:994-1004.

38 Wapinski OL, Vierbuchen T, Qu K, et al. Hierarchical mechanisms for direct reprogramming of fibroblasts to neurons. Cell 2013; 155:621-635.

39 Soufi A, Garcia MF, Jaroszewicz A, Osman N, Pellegrini M, Zaret KS. Pioneer transcription factors target partial DNA motifs on nucleosomes to initiate reprogramming. Cell 2015; 161:555-568.

40 Krizhanovsky V, Lowe SW. Stem cells: the promises and perils of p53. Nature 2009; 460:1085-1086.

41 Koche RP, Smith ZD, Adli M, et al. Reprogramming factor expression initiates widespread targeted chromatin remodeling. Cell Stem Cell 2011; 8:96-105.

42 Rais Y, Zviran A, Geula S, et al. Deterministic direct reprogramming of somatic cells to pluripotency. Nature 2013; 502:65-70.

43 Wilson BG, Wang X, Shen X, et al. Epigenetic antagonism between polycomb and SWI/SNF complexes during oncogenic transformation. Cancer Cell 2010; 18:316-328.

44 Harris SL, Levine AJ. The p53 pathway: positive and negative feedback loops. Oncogene 2005; 24:2899-2908.

45 Bakkenist CJ, Kastan MB. DNA damage activates ATM through intermolecular autophosphorylation and dimer dissociation. Nature 2003; 421:499-506.

46 Oh J, Sohn DH, Ko M, et al. BAF60a interacts with p53 to recruit the SWI/SNF complex. J Biol Chem 2008; 283:1192411934.

47 Zaret K. Micrococcal nuclease analysis of chromatin structure. Curr Protoc Mol Biol 2005 Feb; Chapter 21:Unit 21.1.

48 MacIsaac KD, Lo KA, Gordon W, Motola S, Mazor T, Fraenkel E. A quantitative model of transcriptional regulation reveals the influence of binding location on expression. PLoS Comput Biol 2010; 6:e1000773.

49 Landolin JM, Johnson DS, Trinklein ND, et al. Sequence features that drive human promoter function and tissue specificity. Genome Res 2010; 20:890-898.

50 Zheng R, Rebolledo-Jaramillo B, Zong Y, et al. Function of GATA factors in the adult mouse liver. PLoS One 2013; 8:e83723.

51 Li RX, Chen HB, Tu K, et al. Localized-statistical quantification of human serum proteome associated with type 2 diabetes. PLoS One 2008; 3:e3224.

52 Sanjana NE, Shalem O, Zhang F. Improved vectors and genome-wide libraries for CRISPR screening. Nat Methods 


\section{4; 11:783-784}

(Supplementary information is linked to the online version of the paper on the Cell Research website.)

(c) (i)

\section{(c)} third party material in this article are included in the article's Creative Commons license, unless indicated otherwise in the credit line; if the material is not included under the Creative Commons license, users will need to obtain permission from the license holder to reproduce the material. To view a copy of this license, visit http:// creativecommons.org/licenses/by/4.0/

(C) The Author(s) 2017 